\title{
Clinical and immunological assessment of asymptomatic SARS-CoV-2 infections
}

\author{
Quan-Xin Long ${ }^{1,8}$, Xiao-Jun Tang ${ }^{2,8}$, Qiu-Lin Shi²,8, Qin Liं ${ }^{3,8}$, Hai-Jun Deng ${ }^{1,8}$, Jun Yuan', Jie-Li Hu',

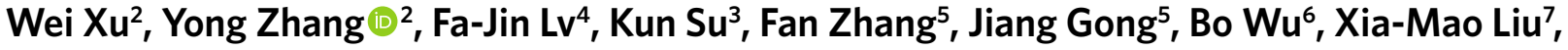 \\ Jin-Jing $\mathrm{Li}^{7}$, Jing-Fu Qiu ${ }^{2}{ }^{凶}$, Juan Chen ${ }^{1 凶}$ and Ai-Long Huang ${ }^{1 凶}$
}

\begin{abstract}
The clinical features and immune responses of asymptomatic individuals infected with severe acute respiratory syndrome coronavirus 2 (SARS-CoV-2) have not been well described. We studied 37 asymptomatic individuals in the Wanzhou District who were diagnosed with RT-PCR-confirmed SARS-CoV-2 infections but without any relevant clinical symptoms in the preceding $14 \mathrm{~d}$ and during hospitalization. Asymptomatic individuals were admitted to the government-designated Wanzhou People's Hospital for centralized isolation in accordance with policy'. The median duration of viral shedding in the asymptomatic group was $19 \mathrm{~d}$ (interquartile range (IQR), 15-26 d). The asymptomatic group had a significantly longer duration of viral shedding than the symptomatic group (log-rank $P=\mathbf{0 . 0 2 8}$ ). The virus-specific IgG levels in the asymptomatic group (median S/CO, 3.4; IQR, 1.6-10.7) were significantly lower $(P=0.005)$ relative to the symptomatic group (median S/CO, 20.5; IQR, 5.8-38.2) in the acute phase. Of asymptomatic individuals, $93.3 \%(28 / 30)$ and $81.1 \%$ $(30 / 37)$ had reduction in IgG and neutralizing antibody levels, respectively, during the early convalescent phase, as compared to $96.8 \%(30 / 31)$ and $62.2 \%(23 / 37)$ of symptomatic patients. Forty percent of asymptomatic individuals became seronegative and $12.9 \%$ of the symptomatic group became negative for IgG in the early convalescent phase. In addition, asymptomatic individuals exhibited lower levels of 18 proand anti-inflammatory cytokines. These data suggest that asymptomatic individuals had a weaker immune response to SARS-CoV-2 infection. The reduction in IgG and neutralizing antibody levels in the early convalescent phase might have implications for immunity strategy and serological surveys.
\end{abstract}

As of May 24, 2020, the coronavirus disease 2019 (COVID-19) pandemic, caused by SARS-CoV-2, has affected more than 5 million people around the world. Most patients with SARS-CoV-2 infections have reportedly had mild to severe respiratory illness with symptoms such as fever, cough and shortness of breath, which might appear 2-14 d after exposure. However, there are other patients who are diagnosed by a positive RT-PCR test but are either asymptomatic or minimally symptomatic ${ }^{2-6}$. Increasing evidence has shown that asymptomatic individuals can spread the virus efficiently, and the emergence of these silent spreaders of SARS-CoV-2 has caused difficulties in the control of the epidemic ${ }^{2,5}$. However, our understanding of the clinical features and immune responses of asymptomatic individuals with SARS-CoV-2 infection is limited. Here we describe the epidemiological and clinical characteristics, virus levels and immune responses in 37 asymptomatic individuals.

\section{Results}

Demographic characteristics. On February 6, 2020, the National Health Commission of China updated the COVID-19 Prevention and Control Plan (4th edition) for the management of close contacts, emphasizing identification and quarantine of asymptomatic individuals ${ }^{1}$. To identify asymptomatic individuals, the Wanzhou District Centers for Disease Control and Prevention (CDC) then conducted extensive RT-PCR screening for 2,088 close contacts under quarantine. Individuals with positive RT-PCR results then were screened by point prevalence surveys carried out by the local CDC and symptoms assessments reported by clinicians. Of these, 60 individuals claimed no symptoms in the preceding $14 \mathrm{~d}$, according to local CDC records, and were transferred to a government-designated hospital for centralized isolation. On admission, 17 individuals were excluded for mild or atypical symptoms based on symptoms assessments reported by clinicians; six individuals who developed symptoms 4-17 d after admission were also excluded. Finally, 37 asymptomatic cases, defined as individuals with a positive nucleic acid test result but without any relevant clinical symptoms in the preceding $14 \mathrm{~d}$ and during hospitalization, were included in this study. A total of 178 patients with confirmed SARS-CoV-2 infections were identified in the Wanzhou District before April 10, 2020, as tracked by CDC surveillance systems. In this study, the proportion of patients with asymptomatic infections was $20.8 \%(37 / 178)$.

For antibody detection and cytokine measurements, 37 sex-, age-frequency- and comorbidity-matched mild symptomatic patients were selected for comparison with the asymptomatic individuals (Supplementary Table 1). Thirty-seven sex- and age-frequency-matched control individuals from the Wanzhou District with negative RT-PCR results for SARS-CoV-2 were also selected for cytokines comparison.

Of the 37 asymptomatic individuals, the median age was 41 years (range, 8-75 years) and 22 were female. Twenty-eight individuals had a confirmed history of contact with an RT-PCR-confirmed patient with COVID-19, and nine were Wuhan residents or had a

\footnotetext{
'Key Laboratory of Molecular Biology on Infectious Diseases, Ministry of Education, Chongqing Medical University, Chongqing, China. ${ }^{2}$ School of Public Health and Management, Chongqing Medical University, Chongqing, China. ${ }^{3}$ Chongqing Center for Disease Control and Prevention, Chongqing, China. ${ }^{4}$ Department of Radiology, The First Affiliated Hospital of Chongqing Medical University, Chongqing, China. ${ }^{5}$ Wanzhou People's Hospital, Chongqing, China. ${ }^{6}$ Wanzhou District Center for Disease Control and Prevention, Chongqing, China. ${ }^{7}$ The Second Affiliated Hospital of Chongqing Medical University, Chongqing, China. ${ }^{8}$ These authors contributed equally: Quan-Xin Long, Xiao-Jun Tang, Qiu-Lin Shi, Qin Li, Hai-Jun Deng. ${ }^{{ }_{e}-m a i l: ~ j f q i u @ 126 . c o m ; ~}$ chenjuan2014@cqmu.edu.cn; ahuang@cqmu.edu.cn
} 


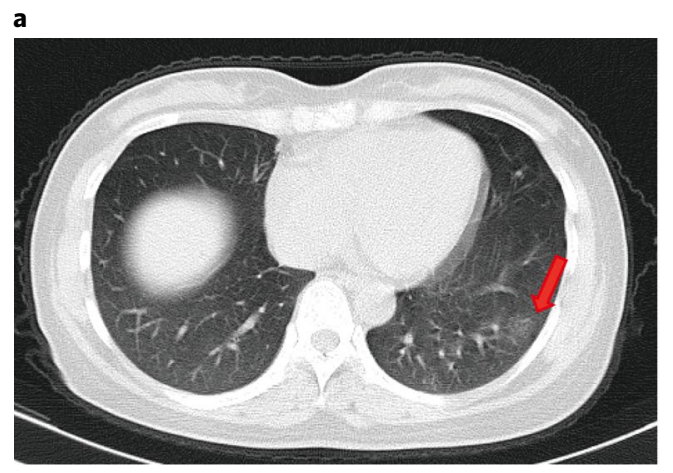

b

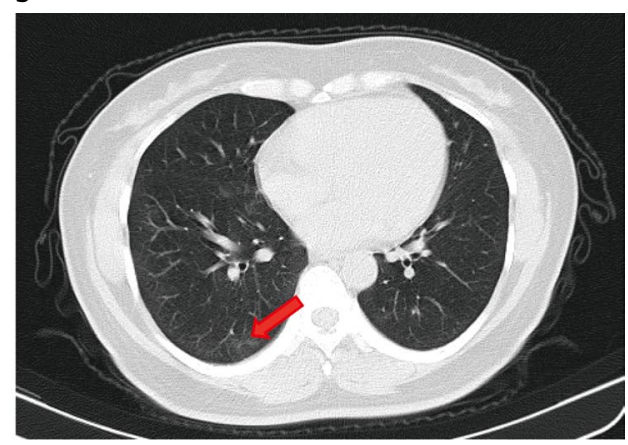

Fig. 1 | Chest CT scans from two asymptomatic patients. a, CT scan of a 45-year-old female showing focal ground-glass opacities in the lower lobe of the left lung (arrow). b, CT scan of a 50-year-old female showing ground-glass opacities and stripes coexisting in the lower lobe of the right lung (arrows).

travel history to Wuhan before the onset of infection (Supplementary Table 2).

Radiologic and laboratory findings. A complete blood count, blood biochemistry, coagulation function, liver and renal function and infection biomarkers were measured upon admission (Supplementary Table 2) to monitor the potential disease progression, according to the COVID-19 Treatment Guidelines (5th edition) from the National Health Commission of $\mathrm{China}^{7}$. Of the 37 asymptomatic individuals, three had lymphopenia and one had thrombocytopenia. Six individuals had elevated levels of alanine aminotransferase, and 11 had increased C-reactive protein levels.

Upon admission, chest computed tomography (CT) scans showed focal ground-glass opacities in 11 asymptomatic individuals $(11 / 37,29.7 \%)$ and stripe shadows and/or diffuse consolidation in ten individuals $(10 / 37,27.0 \%)$, whereas 16 individuals $(16 / 37$, 43.2\%) had no abnormalities (Fig. 1). Five individuals developed focal ground-glass opacities or stripe shadows on chest CT within $5 \mathrm{~d}$ of hospital admission. There were no pleural effusions, air bronchogram signs or enlarged lymph nodes, which were typical changes seen in critically symptomatic patients ${ }^{8-10}$. Abnormal radiological findings confined to one lung were identified in $66.7 \%$ $(14 / 21)$ of the asymptomatic individuals, whereas $33.3 \%(7 / 21)$ had abnormalities in both lungs.

Virological outcomes. We compared the RT-PCR cycle threshold $(\mathrm{Ct})$ values of the first positive nasopharyngeal swabs for all 37 asymptomatic individuals and 37 symptomatic patients. The initial Ct values for 37 asymptomatic individuals and 37 symptomatic patients appeared similar (ORF1b 32.8 (IQR, 30.9-35.8) versus 31.7 (IQR, 30.3-35.1), $P=0.336 ; N 32.6$ (IQR, 29.5-34.6) versus 33.5 (IQR, 31.3-37.2), $P=0.126$ ) (Fig. 2a). The median duration of viral shedding, defined as the interval from the first to last positive nasopharyngeal swab, in the asymptomatic individuals was 19 $\mathrm{d}$ (IQR, 15-26 d). The shortest observed duration of viral shedding was $6 \mathrm{~d}$, whereas the longest was $45 \mathrm{~d}$. The median duration of viral shedding was $14 \mathrm{~d}$ (IQR, 9-22 d) in patients with mild symptoms. The asymptomatic group had a significantly longer duration of viral shedding than the symptomatic group (log-rank $P=0.028$ ) (Fig. 2b). However, measurable virus shedding does not equate with viral infectivity, and further evaluation is needed to determine the respiratory SARS-CoV-2 viral load that is correlated with culturable virus ${ }^{11}$.

Virus-specific IgG and IgM in asymptomatic individuals. To investigate the acute antibody response to SARS-CoV-2 infection, virus-specific IgG and IgM were measured in serum samples from asymptomatic and symptomatic individuals. In the asymptomatic a
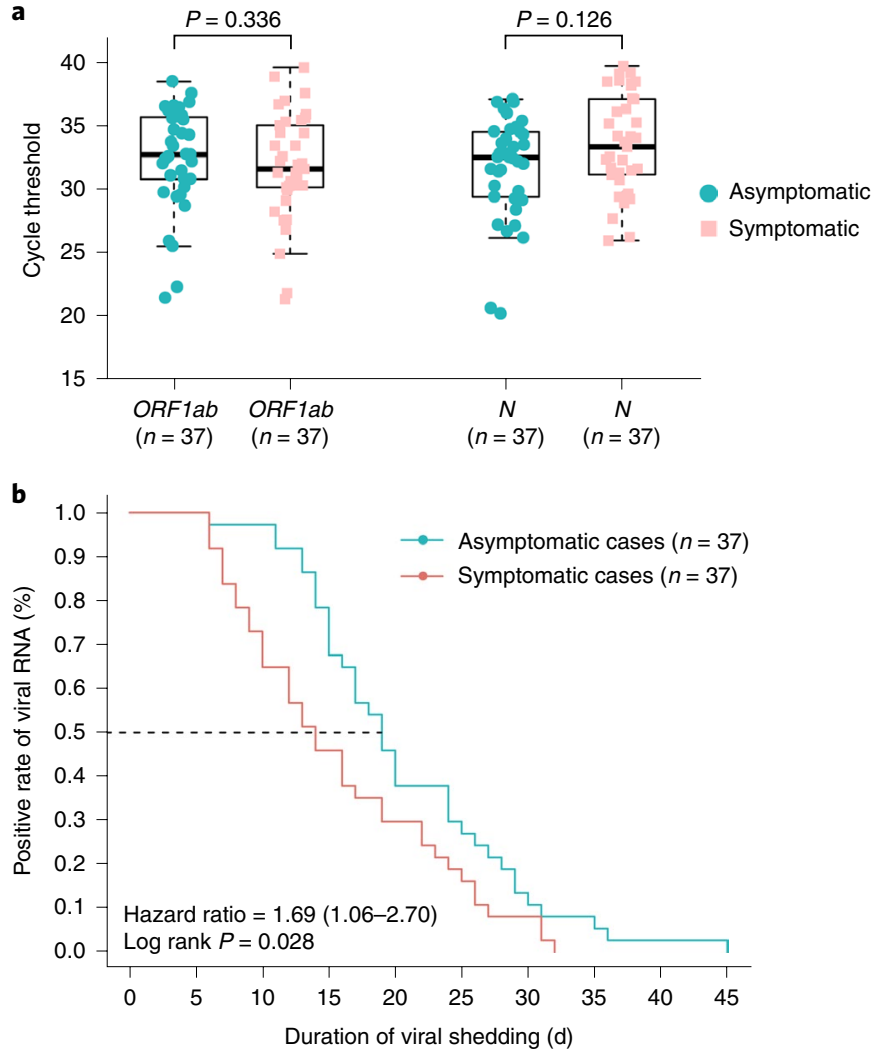

Fig. 2 | Virological characteristics in asymptomatic and symptomatic cases. a, The Ct values of ORF1b and N obtained with RT-PCR that were detected in nasopharyngeal swabs from asymptomatic $(n=37)$ and symptomatic ( $n=37$ ) groups. The box plots show the medians (middle line) and the first and third quartiles (boxes), whereas the whiskers show $1.5 \times$ the IQR above and below the box. Unpaired, two-sided Mann-Whitney $U$ test $P$ values are depicted in the plots, and the significant $P$ value cutoff was set at 0.05. b. The Kaplan-Meier method was used to estimate the positive rate of viral RNA, and the two-sided log-rank test was applied to evaluate the significance difference of the duration of viral shedding in the symptomatic and asymptomatic groups.

group, $81.1 \%$ (30/37) tested positive for $\mathrm{IgG}$, and $83.8 \%(31 / 37)$ of the symptomatic group tested positive for IgG approximately 3-4 weeks after exposure. Moreover, 62.2\% (23/37) of the asymptomatic group were positive for IgM, whereas $78.4 \%$ (29/37) of the symptomatic group were IgM positive. Interestingly, IgG levels in the 

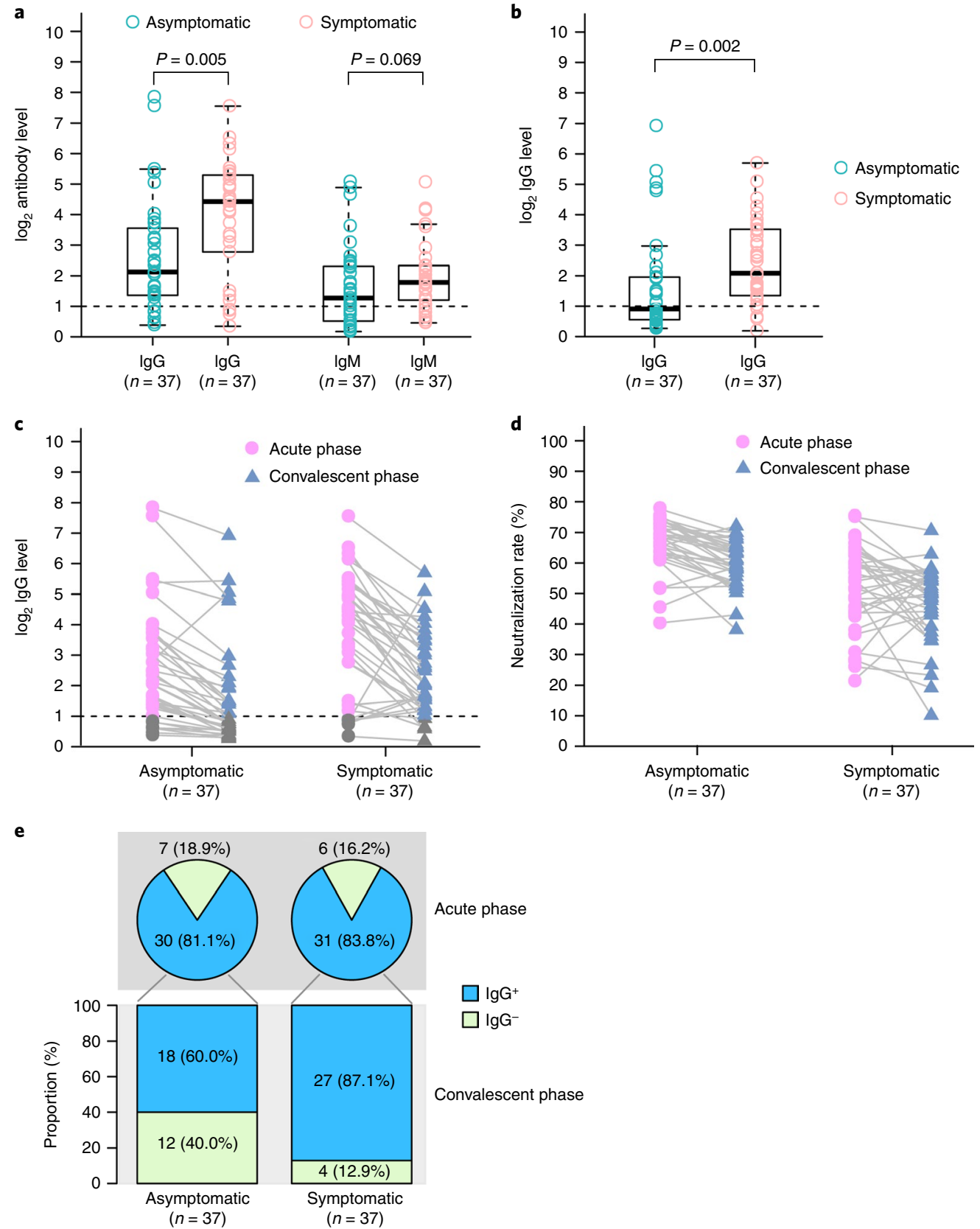

Fig. 3 | lgG and IgM levels in the acute and convalescent phases in patients infected with SARS-CoV-2. a, The comparison of virus-specific antibody levels in asymptomatic patients $(n=37)$ and symptomatic patients $(n=37)$ with acute infections. $\mathbf{b}$, IgG levels in patients with convalescent-phase COVID-19 who were discharged from the hospital. c, Dynamic changes in virus-specific lgG levels in the acute and convalescent phases. d, Dynamic changes in neutralizing serum antibodies in the acute and convalescent phases. Results are expressed as the average of two independent experiments. e, IgG-positive proportions of patients with COVID-19 in the acute and convalescent phases. The box plots in $\mathbf{a}$ and $\mathbf{b}$ show the medians (middle line) and first and third quartiles (boxes), and the whiskers show 1.5x the IQR above and below the box. Unpaired, two-sided Mann-Whitney U test $P$ values are depicted in the plots, and the significant $P$ value cutoff was set at 0.05 .

symptomatic group (median S/CO, 20.5; IQR, 5.8-38.2) were significantly higher than those in the asymptomatic group (median $\mathrm{S} /$ CO, 3.4; IQR, 1.6-10.7) in the acute phase (the period when the viral RNA can be found in a respiratory specimen) $(P=0.005)$ (Fig. 3a).

We also followed 37 asymptomatic individuals and 37 symptomatic patients into the early convalescent phase ( 8 weeks after they were discharged from the hospital). The IgG levels in the symptomatic group were still significantly higher than those in the asymptomatic group in the early convalescent phase $(P=0.002)$ (Fig. 3b). Surprisingly, the IgG levels in $93.3 \%$ (28/30) of the asymptomatic group and $96.8 \%(30 / 31)$ of the symptomatic group declined dur- ing the early convalescent phase (Fig. 3c). The median percentage of decrease was $71.1 \%$ (range, $32.8-88.8 \%$ ) for IgG levels in the asymptomatic group, whereas the median percentage of decrease was $76.2 \%$ (range, $10.9-96.2 \%$ ) in the symptomatic group. Using a pseudovirus-based neutralization assay (Methods), we also observed a decrease in neutralizing serum antibodies levels in $81.1 \%(30 / 37)$ of the asymptomatic group and in $62.2 \%$ (23/37) of the symptomatic group. The median percentage of decrease was $8.3 \%$ (range, $0.5-22.8 \%$ ) for neutralizing serum antibodies in the asymptomatic group, whereas the median percentage of decrease was $11.7 \%$ (range, 2.3-41.1\%) in the symptomatic group (Fig. 3d). Moreover, 

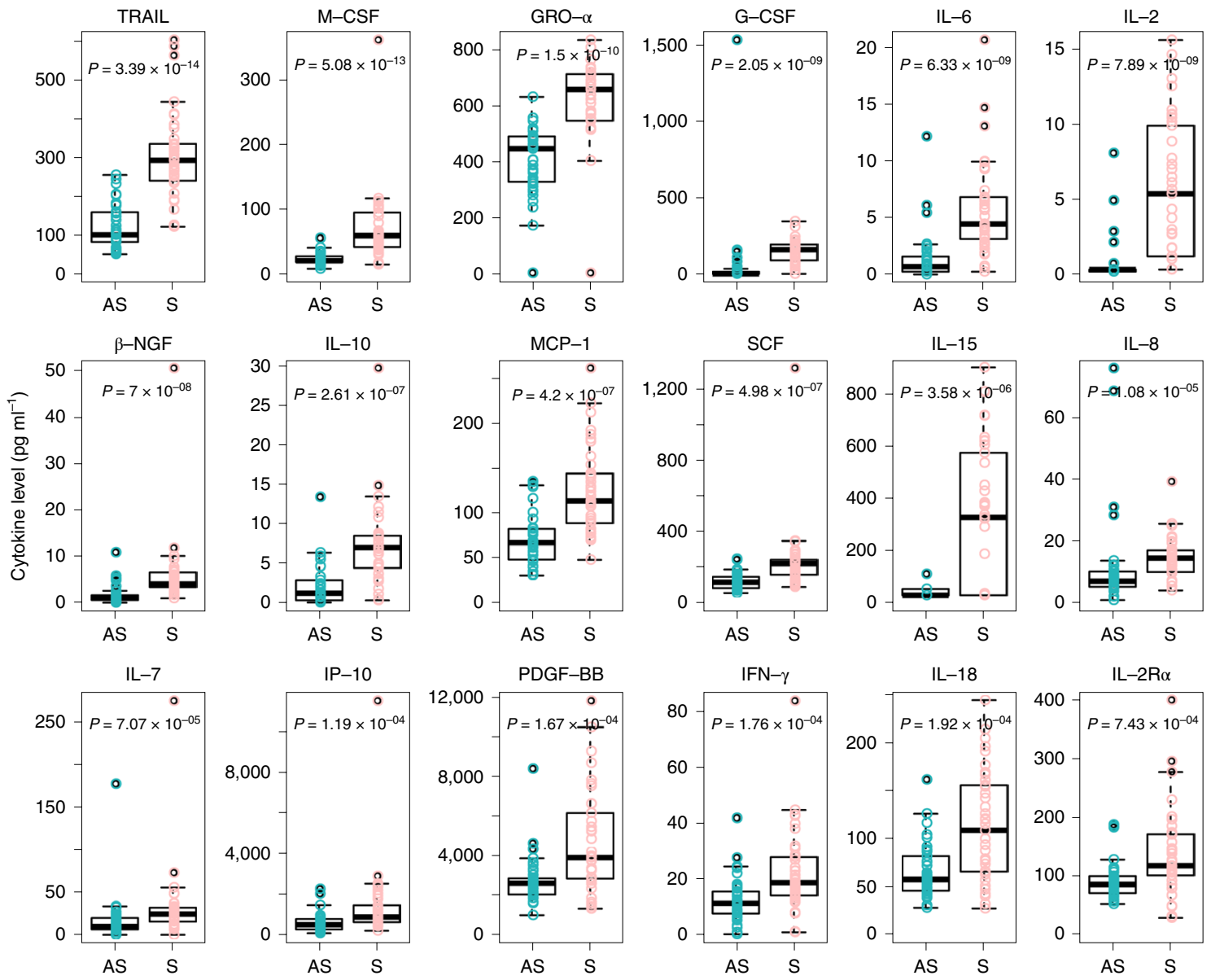

Fig. 4 | Comparison of serum cytokine/chemokine concentrations between the asymptomatic and symptomatic groups. Samples from asymptomatic $(n=37)$ and symptomatic $(n=37)$ patients with COVID-19 were collected in the acute phase during hospitalization, and assays were performed to measure the concentrations of 48 cytokines and chemokines. The box plots show the medians (middle line) and first and third quartiles (boxes), and the whiskers show $1.5 \times$ the IQR above and below the box. Unpaired, two-sided Mann-Whitney $U$ test $P$ values are depicted in the plots, and the significant $P$ value cutoff was set at 0.001 .

$40.0 \%(12 / 30)$ of asymptomatic individuals, but only $12.9 \%$ $(4 / 31)$ of symptomatic individuals, became seronegative for IgG (Fig. 3e).

Cytokines in asymptomatic individuals. To further elucidate the immune responses associated with SARS-CoV-2 infection, serum cytokines and chemokines levels were compared between the asymptomatic and symptomatic groups. Elevated concentrations of 18 pro- and anti-inflammatory cytokines were observed in the symptomatic group as compared to the asymptomatic group. Of these, tumor necrosis factor-related apoptosis-inducing ligand (TRAIL) $\left(P=3.39 \times 10^{-14}\right)$, macrophage colony-stimulating factor $(\mathrm{M}-\mathrm{CSF})\left(P=5.08 \times 10^{-13}\right)$, growth-regulated oncogene- $\alpha(\mathrm{GRO}-\alpha)$ $\left(P=1.5 \times 10^{-10}\right)$, granulocyte colony-stimulating factor (G-CSF) $\left(P=2.05 \times 10^{-9}\right)$ and interleukin $6\left(\right.$ IL-6) $\left(P=6.33 \times 10^{-9}\right)$ showed the most significant changes (Fig. 4 and Extended Data Fig. 1). Moreover, the cytokines were further analyzed in the asymptomatic group and the 37 healthy controls. The plasma levels of 32 cytokines were similar between the healthy controls and the asymptomatic individuals. Significantly higher levels of stem cell factor (SCF) $\left(P=1.48 \times 10^{-9}\right)$, IL-13 $\left(P=3.75 \times 10^{-7}\right)$, IL-12 p40 $\left(P=7.08 \times 10^{-6}\right)$ and leukemia inhibitory factor $(\mathrm{LIF})\left(P=1.33 \times 10^{-3}\right)$ were found in the asymptomatic group (Extended Data Fig. 2). Collectively, our data show that the asymptomatic individuals had a reduced inflammatory response characterized by low circulating concentrations of cytokines and chemokines.

\section{Discussion}

The clinical features and immune responses of asymptomatic individuals infected with SARS-CoV-2 have not been well described. Of the 178 laboratory-confirmed patients, 37 who never developed any symptoms throughout the disease course were included in this study. Our data showed that $20.8 \%$ of these patients had asymptomatic infections. However, this might not be an accurate estimation of the proportion of asymptomatic infections in the general population owing to the fact that asymptomatic infections were identified from those who were at high risk for infection (including close contacts and individuals with a history of travel to Wuhan) and not from a random sample of people. Therefore, the proportion of asymptomatic infections needs to be determined through population screening ${ }^{12}$. Additionally, the proportion of asymptomatic infections might be even higher as some cases might be missed by RT-PCR testing. Our group has successfully identified seven patients with SARS-CoV-2 infection from 148 cases with negative RT-PCR results and no symptoms by using an antibody test $^{13}$. Therefore, timely RT-PCR and serological testing should be used in conjunction, which would benefit accurate estimation of the asymptomatic proportion. However, serological testing has limitations, and tests vary in their specificity and sensitivity. Results might also be confounded by previously existing antibodies to SARS-CoV, MERS-CoV or common cold coronaviruses.

To date, the duration of SARS-CoV-2 RNA shedding has not been well characterized. In SARS-CoV, viral RNA was detectable 
in different specimens, including throat swabs, stool and urine, in more than $30 \%$ of patients for as long as 4 weeks after disease onset $^{14}$. In MERS-CoV infections, viral shedding in respiratory secretions persisted for at least 3 weeks ${ }^{15}$. Recently, one study of 191 patients with COVID-19 reported that the median duration of viral shedding was $20 \mathrm{~d}$ in survivors (range, 8-37 d) ${ }^{16}$. In another report, the duration of viral shedding in nasopharyngeal aspirates was prolonged up to at least $24 \mathrm{~d}$ after symptom onset in 18 patients infected with SARS-CoV-2 in Singapore ${ }^{17}$. In this study, the median duration of viral shedding in 37 patients with mild symptoms was $14 \mathrm{~d}$, which was shorter than in previous reports. In comparison to symptomatic patients, the asymptomatic group had a significantly longer duration of viral shedding, with a viral shedding time of $19 \mathrm{~d}$. Several factors might contribute to the variation of duration of viral shedding in different studies, including the severity of disease, definition of duration of viral shedding and frequency of specimen collection. Notably, detection of viral RNA does not necessarily mean that infectious virus is present in respiratory specimens, and caution is required when applying virus shedding duration that was calculated based on RT-PCR to assess infection potential.

The strength and duration of immunity after infection are key issues for 'shield immunity' ${ }^{18}$ and for informing decisions on how and when to ease physical distancing restrictions ${ }^{19,20}$. Previous studies have shown that circulating antibodies against SARS-CoV or MERS-CoV last for at least 1 year ${ }^{21,22}$. Sustained IgG levels were maintained for more than 2 years after SARS-CoV infection ${ }^{23,24}$ Antibody responses in individuals with laboratory-confirmed MERS-CoV infection lasted for at least 34 months after the outbreak $^{25}$. Recently, several studies characterizing adaptive immune responses to SARS-CoV-2 infection have reported that most COVID-19 convalescent individuals have detectable neutralizing antibodies, which correlate with the numbers of virus-specific $\mathrm{T}$ cells ${ }^{26-29}$. In this study, we observed that IgG levels and neutralizing antibodies in a high proportion of individuals who recovered from SARS-CoV-2 infection start to decrease within 2-3 months after infection. In another analysis of the dynamics of neutralizing antibody titers in eight convalescent patients with COVID-19, four patients showed decreased neutralizing antibodies approximately 6-7 weeks after illness onset ${ }^{30}$. One mathematical model also suggests a short duration of immunity after SARS-CoV-2 infection ${ }^{31}$. Together, these data might indicate the risks of using COVID-19 'immunity passports' and support the prolongation of public health interventions, including social distancing, hygiene, isolation of high-risk groups and widespread testing. Additional longitudinal serological studies profiling more symptomatic and asymptomatic individuals are urgently needed to determine the duration of antibody-mediated immunity. In addition, low levels of anti-viral IgG in asymptomatic patients, who might be more likely to become seronegative, further support the need for timely serosurvey to study the true infection rate.

\section{Online content}

Any methods, additional references, Nature Research reporting summaries, source data, extended data, supplementary information, acknowledgements, peer review information; details of author contributions and competing interests; and statements of data and code availability are available at https://doi.org/10.1038/ s41591-020-0965-6.

Received: 1 May 2020; Accepted: 4 June 2020; Published online: 18 June 2020

\section{References}

1. COVID-19 Prevention and Control Plan, 4th edition (National Health Commission of the People's Republic of China, 2020).
2. $\mathrm{Hu}, \mathrm{Z}$. et al. Clinical characteristics of 24 asymptomatic infections with COVID-19 screened among close contacts in Nanjing, China. Sci. China Life Sci. 63, 706-711 (2020).

3. Chan, J. F. et al. A familial cluster of pneumonia associated with the 2019 novel coronavirus indicating person-to-person transmission: a study of a family cluster. Lancet 395, 514-523 (2020).

4. Nishiura, H. et al. Estimation of the asymptomatic ratio of novel coronavirus infections (COVID-19). Int J. Infect. Dis. 94, 154-155 (2020).

5. Bai, Y., et al. Presumed asymptomatic carrier transmission of COVID-19. JAMA 323, 1406-1407 (2020)

6. Mizumoto, K., Kagaya, K., Zarebski, A. \& Chowell, G. Estimating the asymptomatic proportion of coronavirus disease 2019 (COVID-19) cases on board the Diamond Princess cruise ship, Yokohama, Japan, 2020. Euro. Surveill. 25, 2000180 (2020).

7. COVID-19 Treatment Guidelines, 5th edition (National Health Commission of the People's Republic of China, 2020).

8. Xiong, Y. et al. Clinical and high-resolution CT features of the COVID-19 infection: comparison of the initial and follow-up changes. Invest. Radiol. 55, 332-339 (2020)

9. Chen, N. et al. Epidemiological and clinical characteristics of 99 cases of 2019 novel coronavirus pneumonia in Wuhan, China: a descriptive study. Lancet 395, 507-513 (2020).

10. Wang, D., et al. Clinical characteristics of 138 hospitalized patients with 2019 novel coronavirus-infected pneumonia in Wuhan, China. JAMA 323, 1061-1069 (2020).

11. Atkinson, B. \& Petersen, E. SARS-CoV-2 shedding and infectivity. Lancet 395, 1339-1340 (2020).

12. Gudbjartsson, D. F., et al. Spread of SARS-CoV-2 in the Icelandic population N. Engl. J. Med. https://doi.org/10.1056/NEJMoa2006100 (2020).

13. Long, Q. X., et al. Antibody responses to SARS-CoV-2 in patients with COVID-19. Nat. Med. https://doi.org/10.1038/s41591-020-0897-1 (2020).

14. $\mathrm{Xu}, \mathrm{D}$. et al. Persistent shedding of viable SARS-CoV in urine and stool of SARS patients during the convalescent phase. Eur. J. Clin. Microbiol. Infect. Dis. 24, 165-171 (2005).

15. Oh, M. D. et al. Viral load kinetics of MERS coronavirus infection. N. Engl. J. Med. 375, 1303-1305 (2016).

16. Zhou, F. et al. Clinical course and risk factors for mortality of adult inpatients with COVID-19 in Wuhan, China: a retrospective cohort study. Lancet 395, 1054-1062 (2020).

17. Young, B. E., et al. Epidemiologic features and clinical course of patients infected with SARS-CoV-2 in Singapore. JAMA 323, 1488-1494 (2020).

18. Weitz, J. S., et al. Modeling shield immunity to reduce COVID-19 epidemic spread. Nat. Med. https://doi.org/10.1038/s41591-020-0895-3 (2020).

19. Norheim, O. F. Protecting the population with immune individuals. Nat. Med. https://doi.org/10.1038/s41591-020-0895-3 (2020).

20. Phelan, A. L. COVID-19 immunity passports and vaccination certificates: scientific, equitable, and legal challenges. Lancet 395, 1595-1598 (2020).

21. Cao, W. C., Liu, W., Zhang, P. H., Zhang, F. \& Richardus, J. H. Disappearance of antibodies to SARS-associated coronavirus after recovery. N. Engl. J. Med. 357, 1162-1163 (2007).

22. Choe, P. G. et al. MERS-CoV antibody responses 1 year after symptom onset, South Korea, 2015. Emerg. Infect. Dis. 23, 1079-1084 (2017).

23. Guo, X., et al. Long-term persistence of IgG antibodies in SARS-CoV infected healthcare workers. Preprint at https://www.medrxiv.org/content/10.1101/202 $0.02 .12 .20021386 \mathrm{v} 1(2020)$

24. $\mathrm{Wu}, \mathrm{L}$. P. et al. Duration of antibody responses after severe acute respiratory syndrome. Emerg. Infect. Dis. 13, 1562-1564 (2007).

25. Payne, D. C. et al. Persistence of antibodies against middle east respiratory syndrome coronavirus. Emerg. Infect. Dis. 22, 1824-1826 (2016).

26. Ni, L., et al. Detection of SARS-CoV-2-specific humoral and cellular immunity in COVID-19 convalescent individuals. Immunity https://doi.org/10.1101/2020.03.17.20037713 (2020).

27. Thevarajan, I. et al. Breadth of concomitant immune responses prior to patient recovery: a case report of non-severe COVID-19. Nat. Med. 26, 453-455 (2020)

28. $\mathrm{Wu}, \mathrm{F}$, et al. Neutralizing antibody responses to SARS-CoV-2 in a COVID-19 recovered patient cohort and their implications. Preprint at https://www. medrxiv.org/content/10.1101/2020.03.30.20047365v2 (2020).

29. Suthar, M. S., et al. Rapid generation of neutralizing antibody responses in COVID-19 patients. Preprint at https://www.medrxiv.org/content/10.1101/202 0.05.03.20084442v1 (2020).

30. Wang, X., et al. Neutralizing antibodies responses to SARS-CoV-2 in COVID-19 inpatients and convalescent patients. Preprint at https://www. medrxiv.org/content/10.1101/2020.04.15.20065623v3 (2020).

31. Kissler, S. M., Tedijanto, C., Goldstein, E., Grad, Y. H. \& Lipsitch, M. Projecting the transmission dynamics of SARS-CoV-2 through the postpandemic period. Science 368, 860-868 (2020).

Publisher's note Springer Nature remains neutral with regard to jurisdictional claims in published maps and institutional affiliations.

(c) The Author(s), under exclusive licence to Springer Nature America, Inc. 2020 


\section{Methods}

Study design and participants. Between January 21 and February 19, 2020, 25 imported symptomatic patients (registered in Wanzhou District), who returned from Wuhan City or Hubei Province, were confirmed with SARS-CoV-2 infection based on a positive RT-PCR test in Wanzhou District. (Wanzhou is the hardest-hit region of Chongqing City, which is a province-level municipality adjacent to Hubei Province.) Up to April 10, 2020, a total of 2,088 individuals, including close contacts of confirmed patients and people returning from Wuhan, were placed under quarantine by the local CDC and tested by RT-PCR. Of these 2,088 individuals, 93 developed symptoms and tested positive for SARS-CoV-2.

On February 6, 2020, the National Health Commission of China updated the COVID-19 Prevention and Control Plan (4th edition) for the management of close contacts, emphasizing the identification and quarantine of asymptomatic individuals. The local CDC then conducted extensive RT-PCR screening of quarantined individuals. Once a case was confirmed positive by RT-PCR, the first prevalence survey was carried out by the local CDC staff. Individuals confirmed to have COVID-19 were asked to provide information, including demography (data of birth, gender and renal disease), preexisting conditions (including history of hypertension, diabetes mellitus, cardiovascular disease, cerebrovascular disease, chronic lung disease, renal disease, chronic hepatopathy and immunodeficiency diseases) and symptoms, as well as screening records for the preceding $14 \mathrm{~d}$ (including fever, cough, expectoration, shortness of breath, chill, myalgia, sore throat, runny nose, chest distress, headache, diarrhea, vomiting and nausea). According to the COVID-19 Prevention and Control Plan (4th edition), 60 asymptomatic individuals based on preliminary screening were identified and transferred to a government-designated hospital for centralized isolation. On admission, one-on-one interviews were conducted by clinicians to corroborate the asymptomatic claims. After this screening, 17 individuals were excluded for mild or atypical symptoms. Clinicians conduct daily symptom screening once an individual is admitted to the hospital. Four to 17 days after admission, six of these 43 previously asymptomatic individuals developed symptoms and were re-categorized as symptomatic. Finally, 37 individuals with asymptomatic infections were informed about the study and consented to be included in this study.

It was unclear whether individuals who present with asymptomatic infection might progress to clinical disease during the early phase of the SARS-CoV-2 epidemic in China. Therefore, Chinese clinicians chose to administer interferon-alpha (IFN- $\alpha$ ) inhalation, antiviral treatment (ribavirin orally) and supportive treatment (treatment to strengthen immunity, such as thymopentin and Chinese medicine) to avoid possible aggravation, according to the COVID19 Treatment Guidelines (5th edition) from the National Institutes of Health of China, published on February 8, 2020. The isolation and treatment strategy of asymptomatic infections should be updated according to official guidelines in different countries.

Then, 37 sex-, age-frequency- and comorbidity-matched symptomatic patients were selected for comparison with the asymptomatic individuals (Supplementary Table 1). Thirty-seven sex- and age-frequency-matched controls with negative RT-PCR results for SARS-CoV-2 were also included in this study. Individuals with lung, liver, kidney, cardiovascular, metabolic or immunodeficiency diseases were excluded.

Data collection. Epidemiologic, demographic, contact and exposure history, clinical presentations, chest CT, laboratory tests, treatment and outcome data were collected from inpatient medical records. Laboratory data collected for each patient included complete blood count, coagulation profile, serum biochemical tests (including renal and liver function, electrolytes, lactate dehydrogenase and creatine kinase), serum ferritin and biomarkers of infection. Chest CT scans were done for all inpatients.

To identify SARS-CoV-2 infection, nasopharyngeal swabs were collected at least twice and tested by RT-PCR. RNA from all samples was isolated within $24 \mathrm{~h}$. Viral RNA samples were extracted according to manufacturer instructions using the Nucleotide Acid Extraction Kit (DAAN Gene, registration no. 20170583), which based on an automated magnetic bead purification procedure. A commercial RT-PCR kit (DAAN Gene, registration no. 20203400063) was used for testing samples for SARS-CoV-2. Briefly, two target genes, including open reading framelab $(O R F 1 a b)$ and nucleocapsid protein $(N)$, were simultaneously amplified and tested during RT-PCR. Primers of RT-PCR testing for SARS-CoV-2 were according to the recommendation by the Chinese CDC (ORFlab forward: CCCTGTGGGTTTTACACTTAA, ORF1ab reverse: ACGATTGTGCATCAGCT GA, ORF1ab probe: 5' -CCGTCTGCGGTATGTGGAAAGGTTATGG-3' (FAM dye labeled); $N$ forward: GGGGAACTTCTCCTGCTAGAAT, $N$ reverse: CAGACATT.

TTGCTCTCAAGCTG, $N$ probe: $5^{\prime}$-TTGCTGCTGCTTGACAGATT-3' (VIC dye labeled)). PCR cycling: $50^{\circ} \mathrm{C}$ for $15 \mathrm{~min}, 95^{\circ} \mathrm{C}$ for $15 \mathrm{~min}, 45$ cycles containing $94^{\circ} \mathrm{C}$ for $15 \mathrm{~s}, 55^{\circ} \mathrm{C}$ for $45 \mathrm{~s}$ (fluorescence collection).

Ct values less than 37 and greater than 40 were defined as positive and negative, respectively, for both genes. Samples with Ct values from 37 to 40 were defined as inconclusive, and a second test was needed. Starting 1 week after admission, nasopharyngeal samples were tested by RT-PCR every $2-3 \mathrm{~d}$ for the remainder of the hospitalization period. Patients with one positive RT-PCR result were defined as patients with SARS-CoV-2 infection. Patients with two consecutive negative RT$\mathrm{PCR}$ results were defined as SARS-CoV-2 negative.

Definitions. A confirmed case of COVID-19 was defined as an individual with nasopharyngeal swabs that were positive for SARS-CoV-2, using laboratory-based PCR. The symptomatic patients were defined as patients with laboratory-confirmed COVID-19 with symptoms such as fever, cough, sore throat and sputum. An asymptomatic case was defined as an individual with a positive nucleic acid test result but without any relevant clinical symptoms in the preceding $14 \mathrm{~d}$ and during hospitalization. A close contact was defined as (1) anyone who had been within approximately 6 feet ( 2 meters) of a person infected with SARS-CoV-2 for longer than $10 \mathrm{~min}$ and (2) those who had direct contact with the infectious secretions of a COVID-19 patient. Close contact can occur while caring for, living with, visiting or sharing a healthcare waiting area or room with patients with COVID-19. The duration of shedding was calculated as the number of days from the first positive nasopharyngeal sample to the last positive sample based on RTPCR testing. The last positive sample was followed by a negative RT-PCR result on two sequential tests.

\section{Detection of IgG and IgM against SARS-CoV-2. IgG and IgM against} SARS-CoV-2 were detected in plasma samples using magnetic chemiluminescence enzyme immunoassay kits (Bioscience), according to the manufacturer's instructions. Briefly, recombinant antigens containing the nucleoprotein and a peptide (LQPELDSFKEELDKYFKNHTSPDVD) from the spike protein of SARS-CoV-2 were immobilized on magnetic particles.

\section{Neutralization detection using pseudovirus neutralizaion assay. A} codon-optimized spike $(S)$ that lacked the C-terminal 19 amino acids was used to generate a luciferase-expressing pseudovirus. The SARS-CoV-2 pseudovirus neutralization assay was carried out on 293T cells expressing ACE2 in a 96-well plate. Paired dilute sera (1:600) from an individual (one serum sample from the acute phase and another serum sample from the convalescent phase) were obtained, equal volumes of SARS-CoV-2 pseudovirus were added and the plates were pre-incubated at $37^{\circ} \mathrm{C}$ for $1 \mathrm{~h}$. Then, 293T cells expressing ACE2 were incubated with $100 \mu \mathrm{l}$ of sera-pseudovirus mixture for $24 \mathrm{~h}$. Three days after infection, the cells were lysed with $30 \mu$ of lysis buffer (Promega) to measure the pseudoviral transduction. Relative luminescence units of luciferase activity were detected using the Luciferase Assay Kit (Promega), according to the manufacturer's instructions. Experiments were repeated twice. The luciferase activity was determined by GloMax Microplate Luminometer (Promega). The neutralization rate $(\%)$ was calculated as following:

$$
\text { Neutralization Rate }(\%)=\frac{\mathrm{RLU}_{\text {pesudovirus }}-\mathrm{RLU}_{\text {pesudovirus with serum }}}{R L U_{\text {pesudovirus }}-\mathrm{RLU}_{\text {blank }}} 100 \%
$$

Cytokines measurement. The sera of patients with laboratory-confirmed SARS-CoV-2 infections (asymptomatic group $n=37$ and symptomatic group $n=37$ ) were collected as early as possible during hospitalization. The sera of healthy individuals $(n=37)$ were included as control groups. The concentrations of 48 cytokines and chemokines were measured using the Bio-Plex Human Cytokine Screening Panel (48-Plex no. 12007283, Bio-Rad) on a Luminex 200 (Luminex Multiplexing Instrument, Merck Millipore) following the manufacturer's instructions. The 48 cytokines screening panel includes: fibroblast growth factor (FGF), eotaxin, granulocyte colony-stimulating factor (G-CSF), granulocyte-macrophage colony-stimulating factor (GM-CSF), interferon-gamma (IFN- $\gamma$ ), interleukin-1 beta (IL-1 $\beta$ ), interleukin 1 receptor antagonist (IL-1RA), interleukin 1 alpha (IL-1 $\alpha$ ), interleukin-2 receptor-alpha (IL-2R $\alpha$ ), interleukin-3 (IL-3), interleukin-12 p40 (IL-12 (p40)), interleukin-16 (IL-16), interleukin-2 (IL-2), interleukin-4 (IL-4), interleukin-5 (IL-5), interleukin-6 (IL-6), interleukin-7 (IL-7), interleukin-8 (IL-8), interleukin-9 (IL-9), growth-regulated oncogene-alpha (GRO- $\alpha$ ), hepatocyte growth factor (HGF), interferon alpha-2 (IFN- $\alpha 2)$, leukemia inhibitory factor (LIF), monocyte-chemotactic protein 3 (MCP-3), interleukin-10 (IL-10), interleukin-12 p70 (IL-12 (p70)), interleukin-13 (IL-13), interleukin-15 (IL-15), interleukin-17A (IL-17A), interferon gamma-inducible protein (IP-10), monocyte chemoattractant protein-1 (MCP-1), monokine induced by gamma interferon (MIG), beta-nerve growth factor ( $\beta$-NGF), stem cell factor (SCF), stem cell growth factor-beta (SCGF- $\beta$ ), stromal cell-derived factor 1 (SDF-1 $\alpha$ ), macrophage inflammatory protein-1 alpha (MIP-1 $\alpha$ ), macrophage inflammatory protein-1 beta (MIP-1 $\beta$ ), platelet-derived growth factor-BB (PDGF-BB), regulated upon activation, normal T cell expressed and presumably secreted (RANTES), tumor necrosis factor alpha (TNF- $\alpha$ ), vascular endothelial growth factor (VEGF), cutaneous T-cell-attracting chemokine (CTACK), macrophage migration inhibitory factor (MIF), TNF-related apoptosis-inducing ligand (TRAIL), interleukin-18 (IL-18), macrophage colony-stimulating factor (M-CSF) and tumor necrosis factor-beta (TNF- $\beta$ ).

Statistical analysis. All continuous characteristics are described as the medians (IQRs), and categorical characteristics are described as numbers (\%). Significant differences of continuous characteristics between asymptomatic and symptomatic 
and asymptomatic and healthy control groups were determined by the MannWhitney U test. Categorical characteristics comparison was performed with Fisher's exact test. $P$ value cutoffs of 0.05 for antibody and 0.001 for cytokines indicated significance. The Kaplan-Meier method was used to analyze the duration of viral shedding in the symptomatic and asymptomatic groups. Statistical analyses were performed using R software (version 3.6.0).

Ethical approval. The study was approved by the Ethics Commission of Chongqing Medical University (reference no. 2020004). Written informed consent for participation in this study was obtained from all adult participants or guardians on behalf of the children enrolled in this study.

Reporting Summary. Further information on research design is available in the Nature Research Reporting Summary linked to this article.

\section{Data availability}

Raw data in this study are provided in the Supplementary Dataset. Additional supporting data are available from the corresponding authors upon reasonable request. All requests for raw and analyzed data and materials will be reviewed by the corresponding authors to verify whether the request is subject to any intellectual property or confidentiality obligations. Source data are provided with this paper.

\section{Acknowledgements}

We thank C.-Y. Yang and B. Anderson for critical review of the manuscript. This study received the following funding: the National Science and Technology Major Project
(2017ZX10202203); the Emergency Project from the Science \& Technology Commission of Chongqing; and the National Natural Science Foundation of China (grant nos. 81871656 and 8181101099$)$. We acknowledge all patients involved in the study.

\section{Author contributions}

Conceptualization: J.-F.Q., J.C. and A.-L.H. Investigation: Q.-X.L., X.-J.T., Q.-L.S., Q.L. and H.-J.D. Writing-original draft: Q.-X.L., H.-J.D. and J.C. Writing-review and editing: Q.-X.L., H.-J.D., J.C., Q.-L.S., J.-F.Q. and A.-L.H. Funding acquisition: J.C. and A.-L.H. Resources: J.-Y., J.-L.H., W.X., Y.Z., F.-J.L., K.S., F.Z., J.G., B.W., X.-M.L. and J.-J.L. Supervision: A.-L.H.

\section{Competing interests}

The authors declare no competing interests.

\section{Additional information}

Extended data is available for this paper at https://doi.org/10.1038/s41591-020-0965-6.

Supplementary information is available for this paper at https://doi.org/10.1038/ s41591-020-0965-6.

Correspondence and requests for materials should be addressed to J.-F.Q., J.C. or A.-L.H.

Peer review information Saheli Sadanand was the primary editor on this article and managed its editorial process and peer review in collaboration with the rest of the editorial team.

Reprints and permissions information is available at www.nature.com/reprints. 


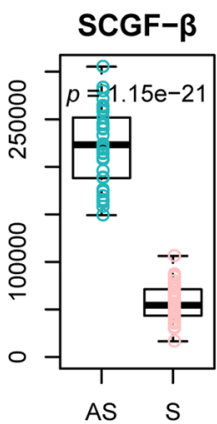

MIG

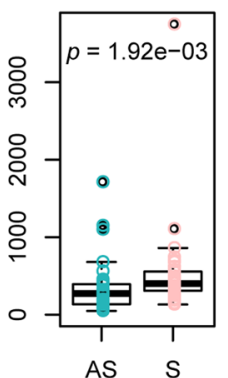

AS $S$

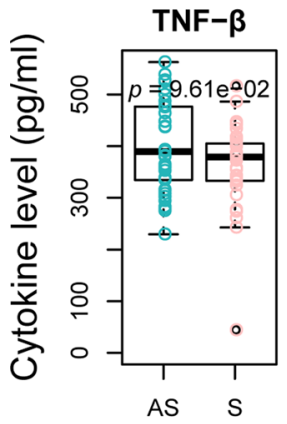

IL-9

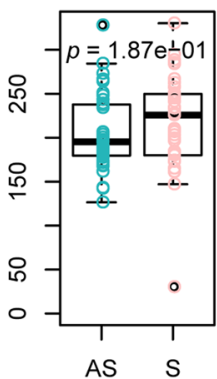

SDF-1a

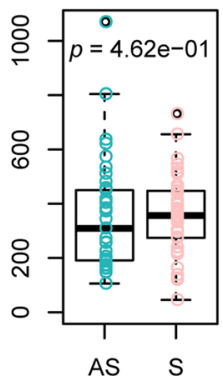

IL-16

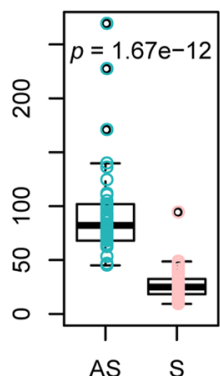

GM-CSF

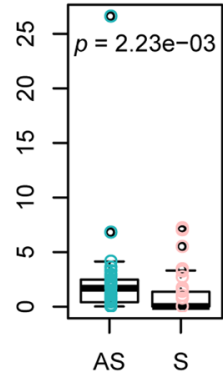

VEGF

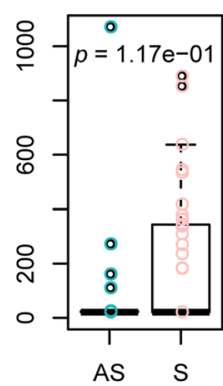

IL-5

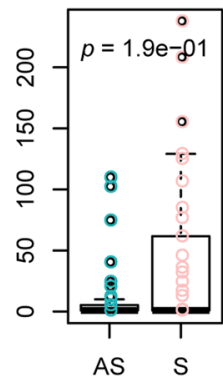

RANTES

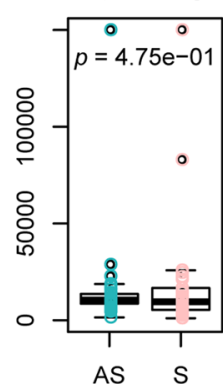

HGF

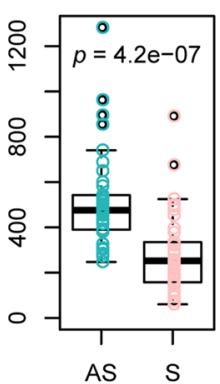

IL-1 $\alpha$
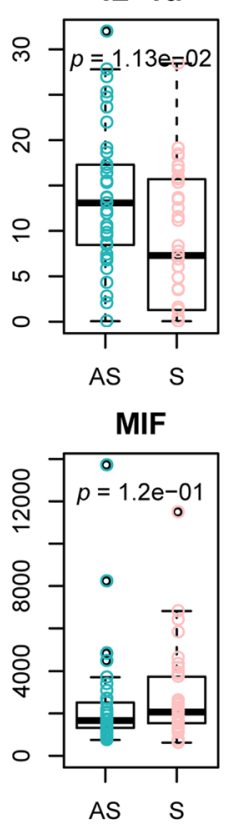

IL-1ß
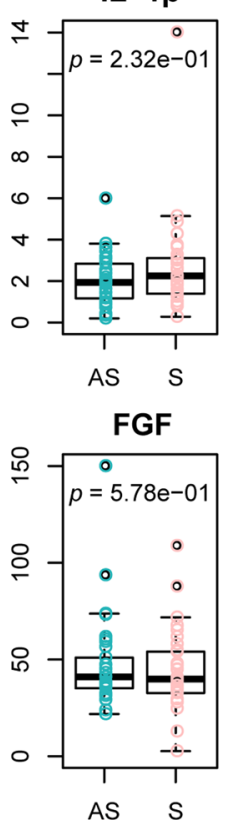

CTACK

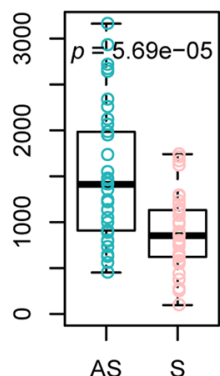

LIF

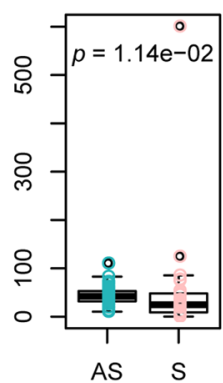

TNF- $\mathbf{a}$

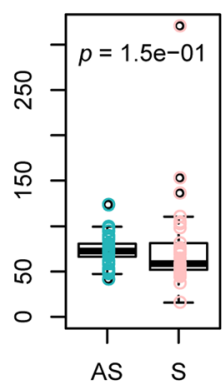

MIP-1a
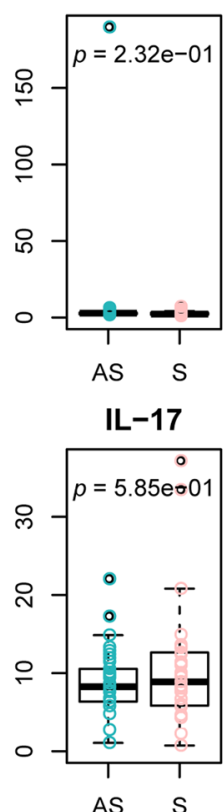

Eotaxin

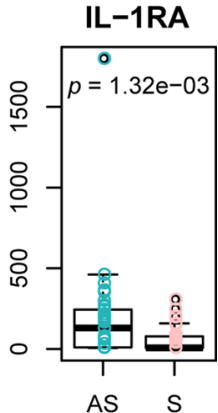

IFN- $\alpha 2$

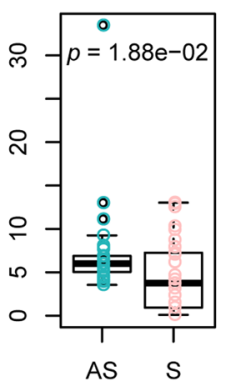

MCP-3
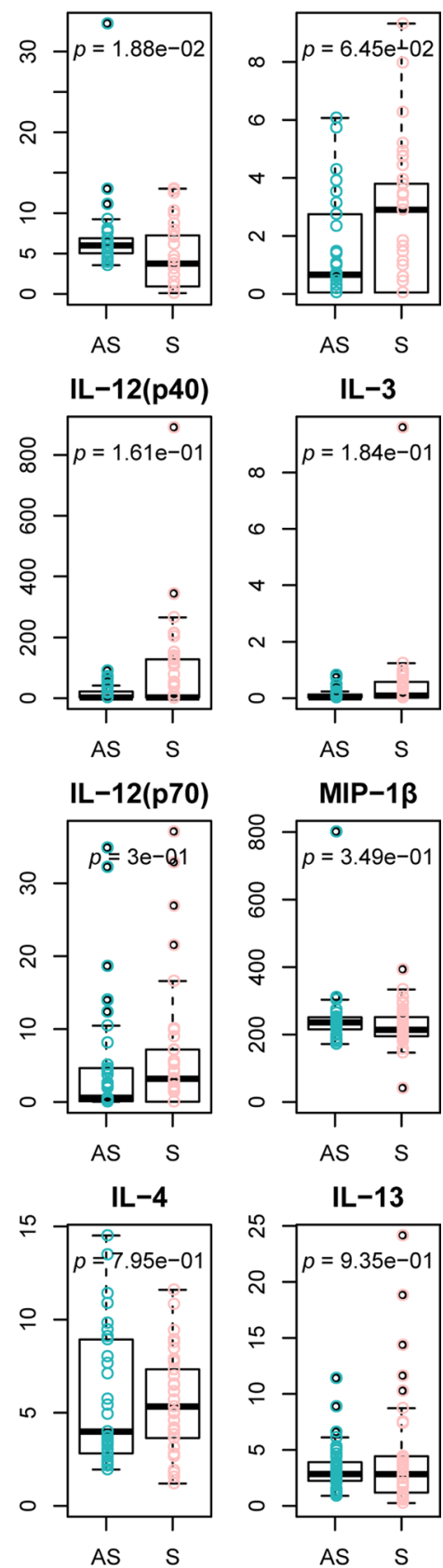

Extended Data Fig. 1 | Comparison of serum cytokine/chemokine concentrations between the asymptomatic and symptomatic groups. Samples from asymptomatic individuals $(n=37)$ and symptomatic patients $(n=37)$ were collected for assays measuring the concentrations of 48 cytokines and chemokines. 25 cytokines or chemokines were similar between asymptomatic individuals and symptomatic patients. 5 cytokines or chemokines were significantly higher in asymptomatic individuals than in symptomatic patients. The boxplots show medians (middle line), first and third quartiles (boxes), while the whiskers show $1.5 \times$ the interquartile range (IQR) above and below the box. Unpaired, two-sided Mann-Whitney $U$ test $p$ values are depicted in plots, and the significant $p$ value cut-off was set to 0.001 . 
A
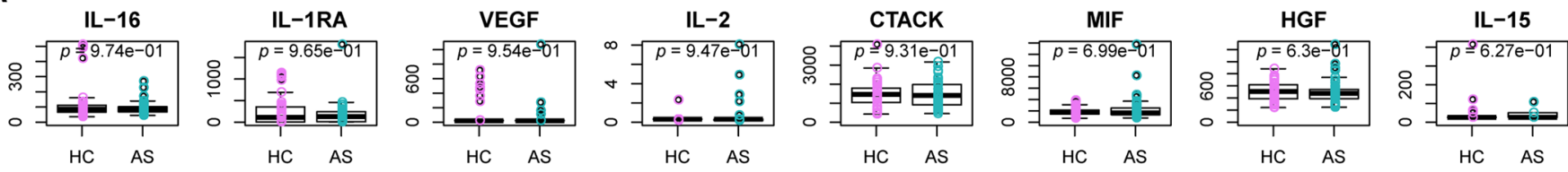

PDGF-BB

IP-10

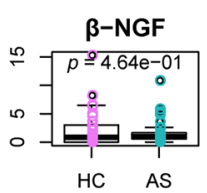

TNF- $\alpha$

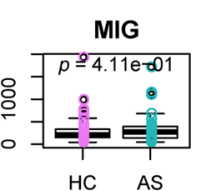

MCP-1

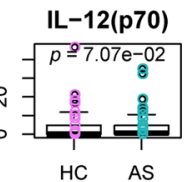

IL-18
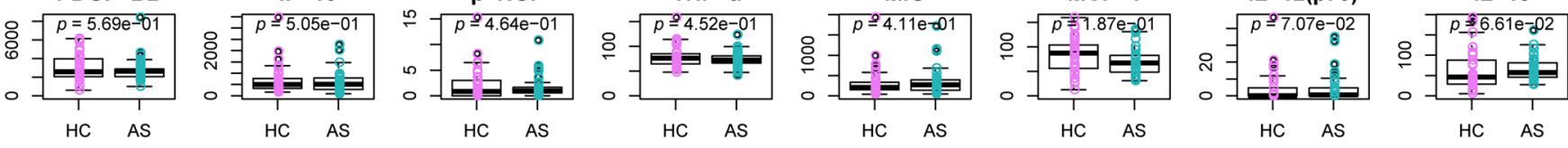

FGF

IL-3

IL-1 $\beta$

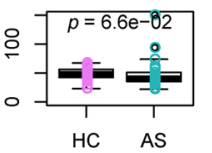

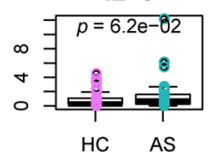
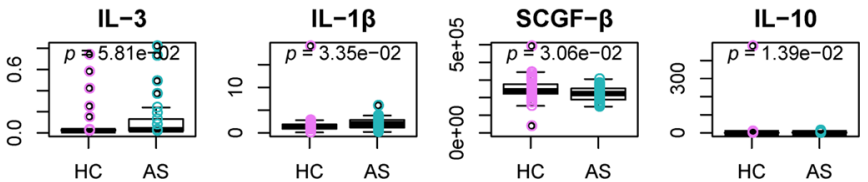

IFN-Y

HC AS

IL-5

M-CSF

TNF- $\beta$

IFN-a2
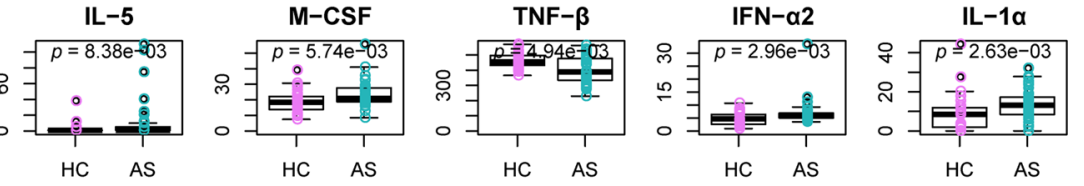

RANTES
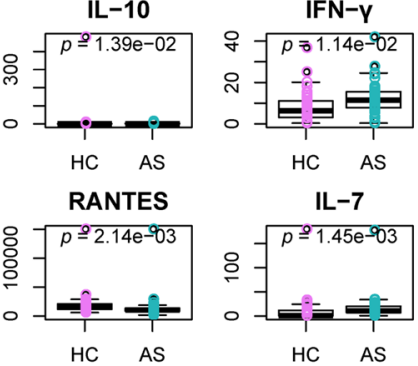

IL-17

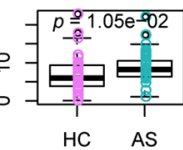

IL-7
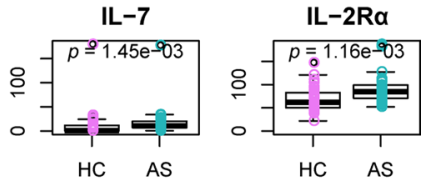

B
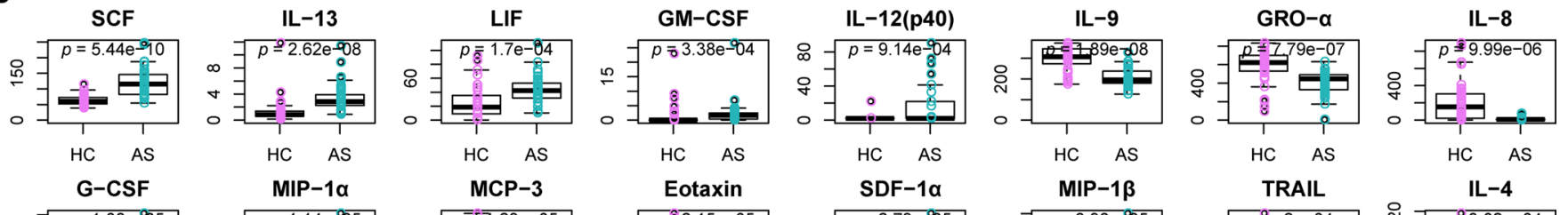

Eotaxin
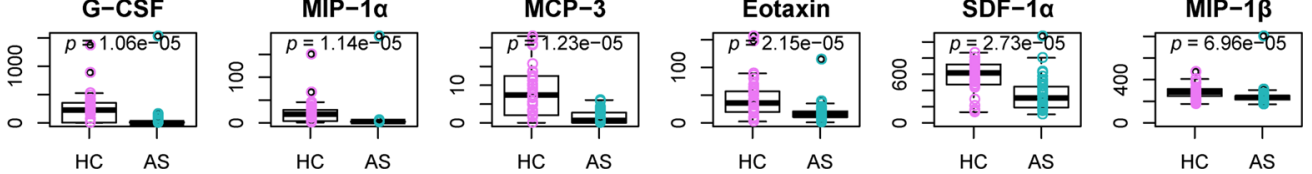

TRAIL
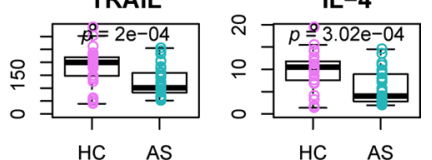

Extended Data Fig. 2 | Comparison of serum cytokine and chemokine concentrations between the asymptomatic and healthy groups. Samples from healthy controls $(n=37)$ and asymptomatic individuals $(n=37)$ were collected for assays measuring the concentrations of 48 cytokines and chemokines. A. Thirty-two cytokines or chemokines were similar between healthy controls and asymptomatic individuals. B. Sixteen cytokines or chemokines were significantly different between healthy controls and asymptomatic individuals. The boxplots show medians (middle line), first and third quartiles (boxes), while the whiskers show $1.5 \times$ the interquartile range (IQR) above and below the box. Unpaired, two-sided Mann-Whitney $U$ test $p$ values are depicted in plots, and the significant $p$ value cut-off was set to 0.001 . 


\section{natureresearch}

Corresponding author(s): Prof. Ai-Long Huang

Last updated by author(s): May 1, 2020

\section{Reporting Summary}

Nature Research wishes to improve the reproducibility of the work that we publish. This form provides structure for consistency and transparency in reporting. For further information on Nature Research policies, see Authors \& Referees and the Editorial Policy Checklist.

\section{Statistics}

For all statistical analyses, confirm that the following items are present in the figure legend, table legend, main text, or Methods section.

$\mathrm{n} / \mathrm{a}$ Confirmed

\ The exact sample size $(n)$ for each experimental group/condition, given as a discrete number and unit of measurement

$\square$ A statement on whether measurements were taken from distinct samples or whether the same sample was measured repeatedly

$\square$ The statistical test(s) used AND whether they are one- or two-sided

Only common tests should be described solely by name; describe more complex techniques in the Methods section.

$\bigotimes \square$ A description of all covariates tested

$\triangle \square$ A description of any assumptions or corrections, such as tests of normality and adjustment for multiple comparisons

A full description of the statistical parameters including central tendency (e.g. means) or other basic estimates (e.g. regression coefficient)

$\triangle$ AND variation (e.g. standard deviation) or associated estimates of uncertainty (e.g. confidence intervals)

For null hypothesis testing, the test statistic (e.g. $F, t, r$ ) with confidence intervals, effect sizes, degrees of freedom and $P$ value noted

Give $P$ values as exact values whenever suitable.

Х $\square$ For Bayesian analysis, information on the choice of priors and Markov chain Monte Carlo settings

Х $\square$ For hierarchical and complex designs, identification of the appropriate level for tests and full reporting of outcomes

$\triangle \square$ Estimates of effect sizes (e.g. Cohen's $d$, Pearson's $r$ ), indicating how they were calculated

Our web collection on statistics for biologists contains articles on many of the points above.

\section{Software and code}

Policy information about availability of computer code

Data collection No commercial, open source software, custom code was used in data collection.

Data analysis Statistical analyses were performed using R software, version 3.6.0.

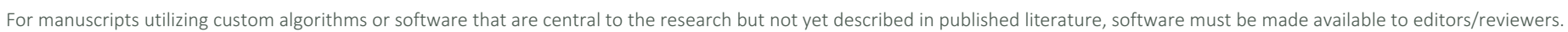
We strongly encourage code deposition in a community repository (e.g. GitHub). See the Nature Research guidelines for submitting code \& software for further information.

\section{Data}

Policy information about availability of data

All manuscripts must include a data availability statement. This statement should provide the following information, where applicable:

- Accession codes, unique identifiers, or web links for publicly available datasets

- A list of figures that have associated raw data

- A description of any restrictions on data availability

Raw data in this study are provided in the Supplementary Dataset. Additional supporting data are available from the corresponding authors on request. All request for raw and analyzed data and materials will be reviewed by the corresponding authors to verify whether the request is subject to any intellectual property or confidentiality obligations. 
Please select the one below that is the best fit for your research. If you are not sure, read the appropriate sections before making your selection.
$\bigotimes$ Life sciences
Behavioural \& social sciences
Ecological, evolutionary \& environmental sciences

For a reference copy of the document with all sections, see nature.com/documents/nr-reporting-summary-flat.pdf

\section{Life sciences study design}

All studies must disclose on these points even when the disclosure is negative.

Sample size There were 178 laboratory-confirmed COVID-19 patients in Wanzhou District (by March 2, 2020), after the symptoms screening, 135 COVID-19 patients were excluded, 6 of the 43 asymptomatic cases developed typical symptoms and were also excluded. The sex- and agefrequency matched controls including 37 symptomatic patients and 37 health subjects were also included in this study.

Data exclusions All patients were included.

Replication

Technical replicates of our assay have been well evaluated. First, the precision and reproducibility of this assay have been evaluated by an independent third-party testing institution (National Institutes for Food and Drug Control), which is required by CFDA Based on the reports given by National Institutes for Food and Drug Control, both the within-run coefficient of variation (CV) and between-run CV were less than $8 \%$, revealing a very good precision and reproducibility of our assay. Second, we also conducted experiments to evaluate the precision and reproducibility of our assay. Thirty serum sample from COVID-19 patients showing different titers of IgG (Range from 0.43 to 187.82) and IgM (Range from 0.26 to 24.02) were tested. Each individual sample was tested in three independent experiments, each involving technical triplicates. Third, we also tested 46 serum sample from COVID-19 patients by using different batches of diagnostic kit for novel coronavirus $\lg$ or $\operatorname{lgM}$ antibody. Very good correlation was found for $\operatorname{lgG}(r=0.996)$ and $\operatorname{lgM}(r=0.997)$. The detailed data has been provided in our previous published paper (QX Long, et al. Antibody responses to SARS-CoV-2 in patients with COVID-19. Nature medicine. 2020 Apr 29. doi: DOI: 10.1038/s41591-020-0897-1; Table 1 and Extend Data Figure 1). After proving the good precision and reproducibility of our assay, the serum sample of each patients was measured once in hospital.

Randomization Our study is an observation study, so no randomization is needed here.

Blinding Serum extraction and antibody detection were performed independently by researchers blind to samples information; data analysis were performed by two trained researchers, and investigators were blinded during data analysis.

\section{Reporting for specific materials, systems and methods}

We require information from authors about some types of materials, experimental systems and methods used in many studies. Here, indicate whether each material, system or method listed is relevant to your study. If you are not sure if a list item applies to your research, read the appropriate section before selecting a response.

\begin{tabular}{l|l} 
Materials \& experimental syster \\
\hline n/a & Involved in the study \\
$\searrow$ & $\square$ Antibodies \\
$\square$ & $\square$ Eukaryotic cell lines \\
$\square$ & $\square$ Palaeontology \\
$\square$ & $\square$ Animals and other organisms \\
$\square$ & $\square$ Clinical data
\end{tabular}

Methods

$\mathrm{n} / \mathrm{a}$ Involved in the study

X $\square$ ChIP-seq

Х $\square$ Flow cytometry

Х $\square$ MRI-based neuroimaging

Eukaryotic cell lines

Policy information about cell lines

Cell line source(s)

293T cell was obtained from ATCC.

Authentication

Authentication was performed regularly based on morphology and gene/protein expression (in case of genetic alterations)

Mycoplasma contamination Cell line was tested for Mycoplasma contamination, no Mycoplasma contamination found.

Commonly misidentified lines (See ICLAC register)

None of the cell line was misidentified in this study. 
Policy information about studies involving human research participants

Population characteristics

For the asymptomatic group, the median age was 41 years, ranged from 8 months to 75 years, and 15 of 37 were male. For the symptomatic group, the median age was 41 ranged from 9 to 75 years, and 19 of 37 were male. For the healthy control group, the median age was 35 ranged from 17 to 72 years, and 16 of 37 were male.

Recruitment

37 individuals with asymptomatic infections without any symptoms in preceding 14 days after exposure and during hospitalization in Wanzhou District were recruited. In order to reduce the confounding bias, 37 sex-, age-frequency and comorbidity-matched symptomatic patients were selected for comparison with the asymptomatic individuals. To minimize selfselection bias, no participants (including asymptomatic and symptomatic patients) had dropped out of this study. In addition, the sex- and age- frequency matched 37 control subjects with negative RT-PCR result for SARS-CoV-2 were also included in this study. Control subjects with lung, liver, kidney, cardiovascular, metabolic or immunodeficiency diseases were excluded. However, the asymptomatic infections were identified from those who were at high risk for infection (including close contacts and individuals with a history of travel to Wuhan) in a single district, not a random sample of people representative of the general population. Therefore, sample selection bias might impact results.

Ethics oversight

The study was approved by the Ethics Commission of Chongqing Medical University (reference number: 2020004). Written informed consent for participation in this study was obtained from all adult participants or guardians on behalf of the children enrolled in this study.

Note that full information on the approval of the study protocol must also be provided in the manuscript. 